\title{
Perspectivas sobre a atuação da Fisioterapia na Saúde Mental
}

\author{
Perspectives on Physical Therapy in Mental Health
}

\author{
Juliana Barbosa Goulardins', Janette Zamudio Canales², Carla Oda ${ }^{3}$ \\ 'Universidade Nove de Julho / Divisão de Fisioterapia, Instituto Central, Hospital das Clínicas HCFMUSP, Faculdade de Medicina, \\ Universidade de Sao Paulo, São Paulo, São Paulo, Brasil. ORCID: 0000-0003-2639-6122. juligoulardins@gmail.com \\ ${ }^{2}$ Instituto de Psiquiatria do Hospital das Clínicas HCFMUSP, Faculdade de Medicina, Universidade de São Paulo. São Paulo, São Paulo, Brasil. \\ ORCID: 0000-0002-6700-1396. janettecanales@gmail.com \\ ${ }^{3}$ Fisioterapeuta. São Paulo, São Paulo, Brasil. ORCID: 0000-0002-6700-1396. carla.oda@uol.com.br
}

Questões de interesse internacional relacionadas à saúde incluem condições frequentes e incapacitantes, como os problemas de saúde mental. Os transtornos mentais têm alta prevalência, estão entre as dez condições que mais causam incapacidade e vem sendo cada vez mais estudados e discutidos por diversos profissionais em todo o mundo. Na infância, os transtornos mentais afetam 10 a $20 \%$ das crianças e adolescentes mundialmente ${ }^{1,2}$. No Brasil, um levantamento recente identificou uma prevalência de transtornos mentais comuns de $30 \%$, entre 74.589 adolescentes residentes em municípios com mais de 100 mil habitantes $^{3}$. Em adultos, constituem a condição com maior índice de "anos perdidos por incapacidade", um indicador que associa o número de anos perdidos por incapacidade com os anos potenciais de vida perdidos em consequência da mortalidade precoce e da carga da condição de saúde ${ }^{4}$.

Os transtornos mentais são caracterizados por alterações clinicamente significativas na cognição, na regulação emocional ou no comportamento do indivíduo, que refletem uma disfunção nos processos psicológicos, biológicos ou de desenvol- vimento subjacentes ao funcionamento mental. Frequentemente são associados a sofrimento ou incapacidade significativos que afetam atividades sociais, profissionais ou outras importantes ${ }^{5}$.

Assim como o conceito de saúde em geral não se restringe a ausência de uma doença, a definição de saúde mental também não se limita a ausência do transtorno mental. Segundo a Organização Mundial da Saúde, a saúde mental compreende "um estado de bem-estar em que o indivíduo realiza suas próprias habilidades, pode lidar com os estresses normais da vida, pode trabalhar de forma produtiva e frutífera, e é capaz de contribuir para a sua comunidade" ${ }^{\prime \prime}$. Os componentes essenciais desta definição (bem-estar, funcionamento efetivo do indivíduo e funcionamento efetivo na comunidade) se assemelham particularmente aos componentes da Classificação Internacional de Funcionalidade (estruturas e funções de corpo, atividades e participação), ferramenta clínica, estatística e de pesquisa amplamente utilizada por fisioterapeutas.

Em todo o mundo, a fisioterapia tem assumido um papel importante, sendo uma opção de tratamento não medicamentoso com destaque na 
prevenção, avaliação e tratamento de diversos problemas de saúde mental, que podem interferir na qualidade de vida e funcionalidade de crianças, jovens, adultos e idosos. A fisioterapia na saúde mental é uma área de atuação que estuda e trata os movimentos e a funcionalidade de pessoas com transtornos mentais e/ou sofrimento psíquico. Tem como objetivo otimizar o bem-estar, promover autocuidado, capacitando o indivíduo através da promoção do movimento funcional, consciência do movimento, atividade física e exercícios e reunindo aspectos físicos, mentais e sociais. Segue a abordagem centrada na pessoa e proporciona cuidados em todos os níveis de atenção à saúde e em todos os ciclos de vida do indivíduo com alterações de saúde mental, sejam elas leves, moderadas ou graves, agudas ou crônicas. Os fisioterapeutas que atuam em saúde mental podem contribuir com equipes multidisciplinares e cuidados inter-profissionais, utilizando-se de abordagens terapêuticas baseadas em evidência? .

Muitos exemplos de como a fisioterapia tem atuado na área de saúde mental podem ser citados, tais como no tratamento da depressão ou da esquizofrenia. Uma metanálise recente identificou efeitos grandes e significativos do exercício aeróbico supervisionado de intensidade moderada em indivíduos com transtorno depressivo maior ${ }^{8}$. O estudo de Dauwan e colaboradores também evidenciou efeitos positivos de qualquer tipo de exercício (aeróbico, fortalecimento, yoga) para sintomas clínicos (total, positivo e negativo), qualidade de vida, função global, depressão e cognição em pessoas com esquizofrenia9. Além disso, diversas técnicas e formas de intervenção que abordam o corpo valorizando aspectos psico-comportamentais têm emergido nos últimos anos, como GDS-Método de Cadeias Musculares e Articulares, Eutonia, Bioenergética, Microfisioterapia, Basic Body Awareness Therapy (BBAT), entre outros. O BBAT, por exemplo, é um programa fisioterapêutico de aprendizado da consciência do movimento, direcionado para a vida diária, coordenação e função ${ }^{10}$, aplicado em diversos transtornos mentais com ótimos resultados ${ }^{11-13}$.

Em alguns municípios brasileiros, os fisioterapeutas já integram as equipes de Saúde Mental dos Centros de Atenção Psicossocial (CAPS) e lá atuam direcionando-se para educação, prevenção e assistência fisioterapêutica de forma individual e coletiva ${ }^{14}$. Embora a atuação já exista em alguns Estados e a disciplina já seja oferecida em algumas universidades, não houve discussão sobre essa nova área de atuação e nem mesmo um consenso sobre o conteúdo a ser abordado nas universidades. Temos aqui um caminho a ser trilhado começando com a formação básica do profissional até práticas de educação permanente.

No entanto, a especialidade Fisioterapia em Saúde Mental ainda não existe no Brasil, mas os esforços estão acontecendo para que essa área de atuação com interface com tantas outras traga muitos benefícios aos pacientes atendidos e amplie de forma massiva 0 mercado para os profissionais.

A fisioterapia aplicada aos transtornos mentais tem um amplo cenário de atuação, sendo eficaz na abordagem de diversas condições, tais como: transtornos do neurodesenvolvimento (ex. transtorno do espectro autista, transtorno do déficit de atenção e hiperatividade, transtorno do desenvolvimento da coordenação, transtorno do desenvolvimento intelectual, etc), transtornos de eliminação (ex. enurese, encoprese), depressão, ansiedade, esquizofrenia, transtornos de sintomas somáticos, disfunções sexuais, transtornos do sono-vigília, demências e outros transtornos cognitivos, dentre outros. Vale ressaltar, que muitas dessas condições já fazem parte da rotina clínica do fisioterapeuta, tanto quando acomete o paciente como diagnóstico principal, quanto como em comorbidade (ex. crianças com paralisia cerebral e autismo ou transtorno do desenvolvimento intelectual; adultos com dores crônicas associadas à depressão ou transtorno de ansiedade, entre outros).

Apesar da forte ligação cientificamente comprovada entre saúde física e mental ${ }^{15}$, a atuação do fisioterapeuta na área de saúde mental ainda é restrita e tema de discussões e opiniões controversas, particularmente no Brasil, cujo principal fator limitador é o desconhecimento. Um ponto a ser esclarecido é a relação entre fisioterapia e saúde mental, que já aparece na própria definição de fisioterapia, de acordo com a Resolução n 80, de 09 de maio de 1987 do Conselho Federal de Fisioterapia e Terapia Ocupacional, "é uma ciência aplicada, cujo objeto de estudos é o movimento humano em todas as suas formas de expressão e potencialidades, quer nas suas alterações patológicas, quer nas suas repercussões psíquicas e orgânicas, com objetivos de preservar, manter, desenvolver ou restaurar a integridade de órgão, sistema ou função". A mesma resolução destaca que como processo terapêutico, o fisioterapeuta utiliza de conhecimentos e recursos próprios, com os quais, baseando-se nas condições psicofísicas e sociais, busca promover, 
aperfeiçoar ou adaptar através de uma relação terapêutica, o indivíduo a uma melhor qualidade de vida.

Alterações de postura e movimento são frequentes em indivíduos com problemas de saúde mental ${ }^{16}$. Em adultos, a prevalência de dores crônicas em indivíduos com transtorno afetivo, ansioso ou relacionado ao estresse excede $30 \%$, e em certas amostras com transtorno de estresse pós-traumático chega a $80 \%{ }^{17}$, condições que impactam diretamente na assistência terapêutica e na forma de prestação de serviços de saúde à população. Estudos têm mostrado que pessoas com problemas de saúde mental são mais suscetíveis à inatividade e estão em risco de um estilo de vida sedentário. Além disso, o uso de drogas psicotrópicas pode resultar no desenvolvimento de síndrome metabólica, obesidade, osteoporose. $\mathrm{Na}$ infância, destaca-se a hipótese de que problemas psicossociais, sintomas depressivos e ansiosos seriam consequências secundárias a problemas moto$\mathrm{res}^{18}$ e tendem a se manifestar muito precocemente, quando a criança é desafiada pelas demandas sociais e por seus pares durante os anos escolares ${ }^{19}$. No entanto, a ocorrência de dificuldades motoras em vários transtornos mentais recebe pouca atenção em comparação aos problemas cognitivos e comportamentais, o que pode impactar negativamente o bem-estar subjetivo, a auto eficácia percebida e a autonomia desses indivíduos. Por exemplo, nos casos de transtorno do déficit de atenção e hiperatividade, existem evidências claras de que 30 a 50\% das crianças em idade escolar com esse diagnóstico apresentam dificuldades motoras, que envolvem alterações de equilíbrio, coordenação motora global e fina ${ }^{20,21}$. Porém, apesar de essas dificuldades serem frequentemente percebidas pelas próprias crianças, com impactos na auto estima (considerada por ela própria e seus pares como "descoordenada", "sempre a última a ser escolhida", dentre outors) e qualidade de vida ${ }^{22}$, os problemas motores ainda recebem pouca atenção na prática clínica23.

Diversos estudos mostram resultados benéficos de técnicas fisioterapêuticas, tais como abordagens corporais (que visam utilizar o corpo como uma forma de trabalhar o movimento, a postura e a expressão emocional ${ }^{24}$ e de atividade física orientada, cinesioterapia, uso de recursos de eletrotermofototerapia (inclusive as técnicas de neuromodulação não invasiva), na melhora de sintomas clínicos de indivíduos com problemas de saúde mental.
Outro ponto a se destacar é que a fisioterapia na saúde mental já é uma área de atuação reconhecida internacionalmente, inclusive como um subgrupo de trabalho da World Confederation of Physical Therapy (WCPT) desde 2011. Além desse marco, outros eventos importantes se destacam na história recente desta área: em 2006, em Leuven na Bélgica, aconteceu a primeira Conferência Internacional de Fisioterapia em Psiquiatria e Saúde Mental, que desde então acontece bianualmente em diferentes países europeus: Noruega, Suécia, Reino Unido, Holanda, Espanha, Islândia, e em 2020 na Finlândia. Em setembro de 2018, na cidade do México, aconteceu o primeiro Congresso de Fisioterapia em Psiquiatria e Saúde Mental do continente americano. Neste evento com a presença de representantes de 15 países, incluindo o presidente da International Organization of Physical Therapy in Mental Health (IOPTHM), Michel Probst, foi assinado um Termo de Cooperação Mútua entre os oito países americanos presentes, sendo eles Brasil, Chile, Colômbia, Costa Rica, Estados Unidos, Equador, Guatemala e México. O objetivo dessa rede é estruturar um plano de trabalho nas áreas de educação, pesquisa e divulgação.

Diante do exposto, fica evidente a importância da atuação do fisioterapeuta em saúde mental e da necessidade do seu reconhecimento como especialidade. Além disso, a adequada habilitação desse profissional é fundamental, através da inclusão dessa temática ao longo de toda sua formação, incluindo princípios básicos desde a graduação, para a sua plena inserção junto à equipe multiprofissional de apoio a esses pacientes.

\section{Agradecimentos}

À Fundação de Amparo à Pesquisa do Estado de São Paulo - FAPESP - Projeto PIPE 2 / ISGAME.

\section{Referências}

1. Belfer ML. Child and adolescent mental disorders: the magnitude of the problem across the globe. J Child Psychol Psychiatry. 2008;49(3):226-36. doi: 10.1111/j.1469$\underline{7610.2007 .01855 . x}$

2. Kieling C, Baker-Henningham H, Belfer M, Conti G, Ertem I, Omigbodun $\mathrm{O}$ et al. Child and adolescent mental health 
worldwide: evidence for action. Lancet. 2011;378(9801):1515-25. doi: 10.1016/S0140-6736(11)60827-1

3. Lopes CS, Abreu GA, Santos DF, Menezes PR, Carvalho KMB, Cunha CF et al. ERICA: prevalence of common mental disorders in Brazilian adolescents. Rev Saúde Pública 2016;50(supl 1):14s. doi: $\underline{10.1590 / \mathrm{s} 01518-8787.2016050006690}$

4. Mathers CD, Loncar D. Projections of global mortality and burden of disease from 2002 to 2030. PLoS Med. 2006;3(11):e442. doi: $10.1371 /$ journal.pmed.0030442

5. American Psychiatric Association. Diagnostic and Statistical Manual of Mental Disorders (DSM-5®). American Psychiatric Pub; 2013.

6. Stenius K. Promoting mental health. concepts, emerging evidence, practice. 2007;102:1989-1991. doi: 10.1111/j.1360$\underline{0443.2007 .02046 . x}$

7. Probst M. Physiotherapy and Mental Health. Clinical Physical Therapy. 2017; 179-204. doi: $\underline{10.5772 / 67595}$

8. Schuch FB, Vancampfort D, Richards J, Rosenbaum S, Ward PB, Stubbs B. Exercise as a treatment for depression: A meta-analysis adjusting for publication bias. J Psychiatr Res. 2016;77:42-51. doi: 10.1016/j.jpsychires.2016.02.023

9. Dauwan M, Begemann MJH, Heringa SM, Sommer IE. Exercise Improves Clinical Symptoms, Quality of Life, Global Functioning, and Depression in Schizophrenia: A Systematic Review and Metaanalysis. Schizophr Bull. 2016;42(3):588-99. doi: 10.1093/schbul/ sbv164

10. Probst M, Skjaerven LH. Physiotherapy in Mental Health and Psychiatry: A Scientific and Clinical Based Approach. Elsevier; 2017.

11. Danielsson L, Rosberg S. Opening toward life: experiences of basic body awareness therapy in persons with major depression. Int J Qual Stud Health Well-being. 2015;10:27069. doi: 10.3402/ ghw.v10.27069

12. Gyllensten AL, Hansson L, Ekdahl C. Outcome of Basic Body Awareness Therapy. A Randomized Controlled Study of Patients in Psychiatric Outpatient Care. Advances in Physiotherapy. 2003;5(4):179-190. doi: 10.1080/14038109310012061

13. Catalan-Matamoros D, Helvik-Skjaerven L, Labajos-

Manzanares MT, Martínez-de-Salazar-Arboleas A, SánchezGuerrero E. A pilot study on the effect of Basic Body Awareness Therapy in patients with eating disorders: a randomized controlled trial. Clin Rehabil. 2011;25(7):617-26. doi:

\section{$10.1177 / 0269215510394223$}

14. Silva SB, Pedrão LJ, Miasso Al. O Impacto da fisioterapia na reabilitação psicossocial de portadores de transtornos mentais. SMAD. 2012;8(1):34-40. doi: 10.11606/issn.1806-6976.v8i1p34-40

15. Ohrnberger J, Fichera E, Sutton M. The relationship between physical and mental health: A mediation analysis. Soc Sci Med.
16. Canales JZ, Fiquer JT, Campos RN, Soeiro-de-Souza MG, Moreno RA. Investigation of associations between recurrence of major depressive disorder and spinal posture alignment: A quantitative cross-sectional study. Gait Posture. 2017;52:258-64. doi: 10.1016/j.gaitpost.2016.12.011

17. Vigo D, Thornicroft G, Atun R. Estimating the true global burden of mental illness. Lancet Psychiatry. 2016;3(2):171-8. doi: 10.1016/S2215-0366(15)00505-2

18. Piek JP, Rigoli D, Pearsall-Jones JG, Martin NC, Hay DA, Bennett KS et al. Depressive Symptomatology in Child and Adolescent Twins With Attention-Deficit Hyperactivity Disorder and/or Developmental Coordination Disorder. Twin Res Hum Genet. 2007;10(04):587-96. doi: 10.1375/twin.10.4.587

19. Piek JP, Bradbury GS, Elsley SC, Tate L. Motor Coordination and Social-Emotional Behaviour in Preschoollaged Children. International Journal of Disability, Development and Education. 2008;55(2):143-51. doi: 10.1080/10349120802033592

20. Goulardins JB, Marques JCB, Casella EB, Nascimento RO, Oliveira JA. Motor profile of children with attention deficit hyperactivity disorder, combined type. Res Dev Disabil. 2013;34(1):40-5. doi: 10.1016/j.ridd.2012.07.014

21. Kaiser ML, Schoemaker MM, Albaret JM, Geuze RH. What is the evidence of impaired motor skills and motor control among children with attention deficit hyperactivity disorder (ADHD)? Systematic review of the literature. Res Dev Disabil. 2015;36C:33857. doi: 10.1016/j.ridd.2014.09.023

22. Goulardins JB, Marques JCFB, Casella EB. Quality of life and psychomotor profile of children with attention deficit hyperactivity disorder (ADHD). Arq Neuropsiquiatr. 2011;69(4):630-5. doi: 10.1590/S0004-282X2011000500011

23. Fliers EA, Franke B, Buitelaar JK. [Motor problems in children with ADHD receive too little attention in clinical practice]. Ned Tijdschr Geneeskd. 2011;155(50):A3559.

24. Young C. The history and development of BodyPsychotherapy: The American legacy of Reich. Body, Movement and Dance in Psychotherapy. 2008;8(1):5-18. doi: $10.1080 / 17432970701717783$ 